\title{
Resonant tunnelling diode based high speed optoelectronic transmitters
}

Jue Wang, G. C. Rodrigues, Abdullah Al-Khalidi, José M. L. Figueiredo, Edward Wasige 


\title{
Resonant Tunnelling Diode Based High Speed Optoelectronic Transmitters
}

\author{
Jue Wang ${ }^{\mathrm{a}}$, G. C. Rodrigues ${ }^{\mathrm{b}}$, Abdullah Al-Khalidi ${ }^{\mathrm{a}}$, José M. L. Figueiredo ${ }^{\mathrm{b}}$ and Edward Wasige ${ }^{\mathrm{a}}$ \\ ${ }^{a}$ School of Engineering, University of Glasgow, Oakfield Avenue, Glasgow, G12 8LT, UK. \\ bDepartamento de Fisica, Universidade do Algarve, Campus de Gambelas, 8005-139 Faro, Portugal.
}

\begin{abstract}
Resonant tunneling diode (RTD) integration with photo detector (PD) from epi-layer design shows great potential for combining terahertz (THz) RTD electronic source with high speed optical modulation. With an optimized layer structure, the RTD-PD presented in the paper shows high stationary responsivity of $5 \mathrm{~A} / \mathrm{W}$ at $1310 \mathrm{~nm}$ wavelength. High power microwave/mm-wave RTD-PD optoelectronic oscillators are proposed. The circuitry employs two RTD-PD devices in parallel. The oscillation frequencies range from $20-44 \mathrm{GHz}$ with maximum attainable power about $1 \mathrm{~mW}$ at 34/37/44GHz.
\end{abstract}

Keywords: resonant tunneling diode, photo diode, oscillator, photodetector

\section{INTRODUCTION}

As the demand for high speed wireless communication continues to increase exponentially, the need to utilize the high speed optic fiber which has been widely employed in most base stations for wireless broadband communications is higher than before. The resonant tunnelling diode photodetector (RTD-PD) technique can provide a low cost solution for the seamless integration of microwave/mm wave radio and fiber networks.

Resonant tunneling diode is considered to be the fastest solid-state electronic device. To date, the highest reported frequency of RTD oscillator is $1.92 \mathrm{THz}$ [1]. To benefit from the RTDs' capability of high frequency sources, the monolithically integration of a light sensitive layer-photo detector was introduced with RTD epi-layer design. RTD based photo-detector shows great potential applications for optical-electrical modulation and photo-detection [3]. Optimization of RTD-PD layer structures to maximize the sensitivity and responsivity is one of the key factors for real applications.

Another key factor is high power/high frequency RTD-PD oscillator design. 30 Gbps wireless communication over a few centimeters distance has been demonstrated by using electrical-only modulated RTD oscillator at $500 \mathrm{GHz}$ [2]. The short distance is mostly due to low output power which was in tens of microwatt range. Different from most RTD relaxation oscillator design [4][5], the proposed RTD-PD LC oscillator circuit utilize two RTD-PDs in parallel. Each device was biased individually to suppress the parasitic oscillation. Device size was also optimized in terms to provide best impedance matching. $1 \mathrm{~mW}$ output power RTD-PD oscillators are reported here. It is expected that with improved device epi-layer design and oscillator output power level, RTD-PD technology can be widely utilized in the long distance/ ultra-high speed optical-wireless communication system.

\section{RTD-PD LAYER STRUCTURE}

As RTD devices exhibit negative differential conductance (NDC) gain over extra wide bandwidth, the RTD-PD device would act as optical receiver with a build in amplifier, which can extract from the optical modulated signal to the RF carriers and amplify it by using RTD as high frequency sources [3][6]. The typical RTD device layer structure consists of a narrow band gap - quantum well material sandwiched between two wide band gap-double barrier materials, namely double barrier quantum well (DBQW) structure. A RTD-PD structure integrates the DBQW within the photo-conductive layers as shown in Table 1, that InGaAs/AlAs RTD was monolithically integrated with $500 \mathrm{~nm}$ photo absorption layerInGaAlAs on top of DBQW. The RTD-PD device is optimized to maximize the optical response, namely the responsivity and sensitivity. Responsivity is a critical parameter to determine the max output saturation power while sensitivity determines the lowest detectable power. The RTD-PD stationary state responsivity $\mathfrak{R}(\lambda, V)$ at bias voltage $V$ is defined *Edward.Wasige@ glasgow.ac.uk, +44 01413308662

Third International Conference on Applications of Optics and Photonics, edited by Manuel F. M. Costa, Proc. of SPIE Vol. 10453, 104532Y · C 2017 SPIE · CCC code: 0277-786X/17/\$18 · doi: 10.1117/12.2276350 
by

$$
\Re_{D C}(\lambda, V)=\frac{I_{\text {light }}(V)-I_{\text {dark }}(V)}{P_{\text {light }}(\lambda)}=M(V) \times \eta_{p h}\left(\frac{e \lambda}{h c}\right)
$$

Where $I_{\text {light }}(V)$ is the current illumination and $I_{\text {dark }}(V)$ is the current under dark conditions, $P_{\text {light }}(\lambda)$ is the incident optical power, $\lambda$ is the wavelength of the incident light wave, $M(V)$ accounts for the internal current gain effect (due to electron-holes generation and/or impact ionization mechanisms), $\eta_{p h}$ is the external quantum efficiency; $e, h$ and $c$ are the elementary charge, the Planck constant and the speed of light in the vacuum, respectively. The built-in gain factor $M(V)$ accounts for the internal current gain effect (due to the photo-generated electron-holes pairs and impact ionization mechanisms). Ideally every photo produces one pair of electron-hole, which contribute to photo current without any loss, the quantum efficiency is equal to one and the ideal responsivity would be given simply by $\mathfrak{R}_{i}=\frac{e \lambda}{h c}$. The ideal maximum responsivity for wavelength $1310 \mathrm{~nm}$ and $1550 \mathrm{~nm}$ is $1.05 \mathrm{~A} / \mathrm{W}$ and $1.25 \mathrm{~A} / \mathrm{W}$. In reality, many factors reduce the ideal responsivity value such as limited optical absorption efficient, finite absorption layer thickness (penetration depth), and optical reflection at the semiconductor surface, losses due to carrier recombination, and also the detector bandwidth limitation. The frequency response of the RTD-PD is not presented in the paper.

Table 1. RTD-PD epi-layer I.

\begin{tabular}{|c|c|c|c|c|}
\hline Layer \# & Thickness $(\AA)$ & Composition & Doping $\left(\mathrm{cm}^{-3}\right)$ & Description \\
\hline 12 & 450 & InGaAs & $3 \mathrm{E} 19: \mathrm{Si}$ & Collector \\
\hline 11 & 800 & InGaAs & $3 \mathrm{E} 18: \mathrm{Si}$ & Collector \\
\hline 10 & 5000 & InGaAs & $5 \mathrm{E} 16: \mathrm{Si}$ & Absorption \\
\hline 9 & 20 & InGaAs & Un-doped & Spacer \\
\hline 8 & 17 & AlAs & Un-doped & Barrier \\
\hline 7 & 47 & InGaAs & Un-doped & Well \\
\hline 6 & 17 & AlAs & Un-doped & Barrier \\
\hline 5 & 20 & InGaAs & InGaAs & Spacer \\
\hline 4 & 200 & InGaAs & 2E16:Si & Spacer \\
\hline 3 & 80 & InAlAs & 3E18 : Si & Emitter \\
\hline 2 & 4000 & InGaAs & 3E19:Si & Emitter \\
\hline 1 & 200 & InP & Un-doped & Buffer \\
\hline \multicolumn{5}{|l|}{ Substrate SI $:$ InP } \\
\hline
\end{tabular}

\subsection{DC characteristics}

In this paper, $10 \times 10 \mu \mathrm{m}^{2}$ sized RTD-PD are investigated. The central optical window is rectangular shape. As shown in Fig 1 (a), the optical window is $3 \times 8 \mu \mathrm{m}^{2}$ for the device design while Fig 1 (b) shows the fabricated device with clearopened optical window. The I-V characteristics under dark and light illumination are compared in Fig 2. Under dark condition, the device shows negative differential conductance (NDC) region between $1.1 \mathrm{~V}-1.4 \mathrm{~V}$. The peak current density was about $0.3 \mathrm{~mA} / \mathrm{um}^{2}$, peak to valley current ratio (PVCR) was about 1.5 . The device stationary optical response was investigated with non-modulated continuous wave $(\mathrm{CW})$ light at $1310 \mathrm{~nm}$ and $1550 \mathrm{~nm}$. The measurement work was done at Universidade do Algarve. The measurement setup is shown in the inset of Fig 2. The RTD-PD was biased from left (probe). The cleaved single mode optical fiber was aligned with the optical window from device top mesa. The light with wavelength $1310 \mathrm{~nm}$ and $1550 \mathrm{~nm}$ was coupled into the RTD-PD with $0.1 \mathrm{~mW}$ and $1 \mathrm{~mW}$ power. As shown in Fig 2. (a), the optical DC response of $1310 \mathrm{~nm}$ laser with different optical power are compared with dark current. The maximum response was about $5 \mathrm{~A} / \mathrm{W}$ when RTD-PD was biased close to the peak voltage and the injected light power was $1 \mathrm{~mW}$. As shown in Fig 2. (b), the maximum responsivity of $1550 \mathrm{~nm}(1 \mathrm{~mW})$ was about $2 \mathrm{~A} / \mathrm{W}$. The main difference is due to high absorption efficiency at $1310 \mathrm{~nm}$ wavelength. 


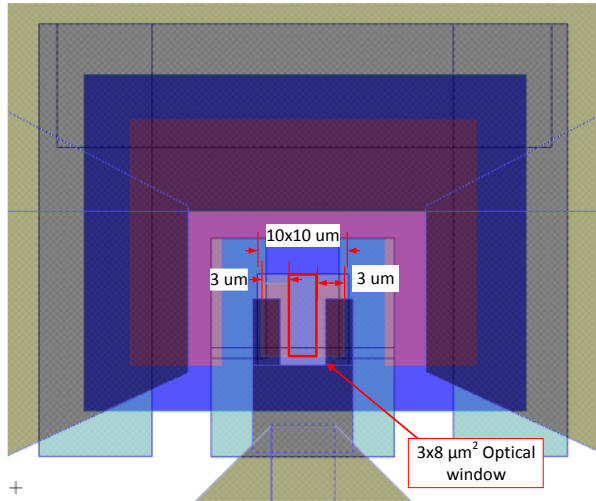

(a)

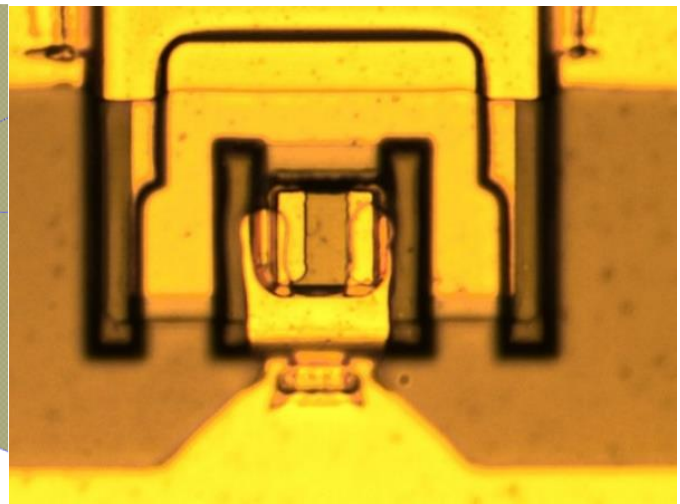

(b)

Figure 1. (a) RTD-PD layout design. The device size was $10 \times 10 \mu \mathrm{m}^{2}$, the optical window size was $3 \times 8 \mu \mathrm{m}^{2}$. (b) The micrograph of the top-view of the fabricated device. The optical window is clearly noted.

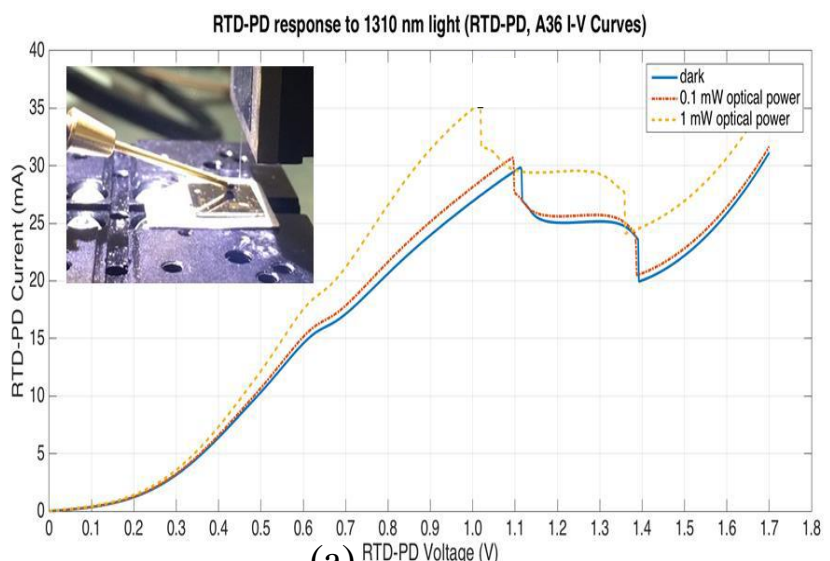

(a)

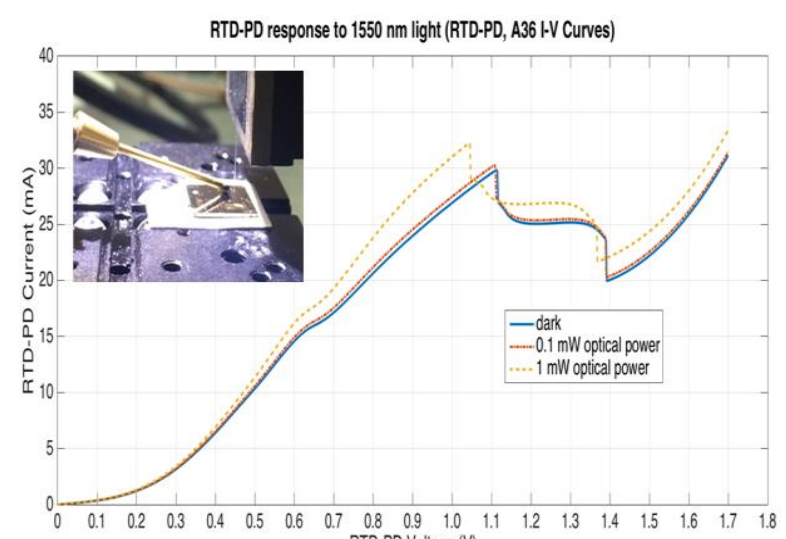

(b)

Figure 2. RTD-PD stationary optical response with light injection power of $0.1 \mathrm{~mW}$ and $1 \mathrm{~mW}$ (a) The optical response of $1310 \mathrm{~nm}$ wavelength. (b) The optical response of $1550 \mathrm{~nm}$ wavelength. The device shows large responsivity of 5 A/W for $1310 \mathrm{~nm}$ compared to $1550 \mathrm{~nm}$ due to high absorption efficiency. Inset shows the measurement setup. The device was biased from left (probe). The injected light was coupled from the top side.

\section{RTD-PD OSCILLATORS}

High frequency RTD-PD oscillator design utilized the device described above. New epi-layer structure including double light absorption layer on both side of DBQW as shown in Table 2 are introduced and characterized for the consideration of power performance and better optical control. As shown in Figure 3, two different sized devices, $10 \times 10 \mu \mathrm{m}^{2}, 15 \times 15$ $\mu \mathrm{m}^{2}$ are investigated. The central optical windows are $3 \times 8 \mu \mathrm{m}^{2}$ and $5 \times 10 \mu \mathrm{m}^{2}$ respectively. The NDC region was between $1.2 \mathrm{~V}-2.4 \mathrm{~V}$. The peak current density was about $0.16 \mathrm{~mA} / \mathrm{um}^{2}$ and peak to valley current ratio (PVCR) was about 2 . The measurement results are summarize in Table 3 , where $\Delta \mathrm{V}$ is the peak-valley voltage difference, $\Delta \mathrm{I}$ is the peak-valley current difference. The maximum negative difference conductance $\mathrm{G}_{\mathrm{n}}$ is given by $\frac{3 \Delta \mathrm{I}}{2 \Delta \mathrm{V}}$. The estimated maximum power for each single device is given by $\frac{3}{16} \Delta V \Delta \mathrm{I}$ when load conductance is $\mathrm{G}_{\mathrm{L}}=\mathrm{G}_{\mathrm{n}} / 2$ [7][8]. Benefit from large $\Delta \mathrm{V}$, these devices shows potential for higher power oscillator design than the above epi-layer I (Table 1) devices. 
Table 2. RTD-PD epi-layer II.

\begin{tabular}{|c|c|c|c|c|}
\hline Layer \# & Thickness $(\AA)$ & Composition & Doping $\left(\mathrm{cm}^{-3}\right)$ & Description \\
\hline 13 & 1000 & InGaAs & $3 \mathrm{E} 19: \mathrm{Si}$ & Collector \\
\hline 12 & 3000 & InAlAs & $3 \mathrm{E} 18: \mathrm{Si}$ & Collector \\
\hline 11 & 5000 & InGaAlAs & $2 \mathrm{E} 16: \mathrm{Si}$ & Absorption \\
\hline 10 & 20 & InGaAlAs & Un-doped & Spacer \\
\hline 9 & 17 & AlAs & Un-doped & Barrier \\
\hline 8 & 57 & InGaAs & Un-doped & Well \\
\hline 7 & 17 & AlAs & Un-doped & Barrier \\
\hline 6 & 20 & InGaAlAs & Un-doped & Spacer \\
\hline 5 & 5000 & InGaAlAs & 2E16 $: S i$ & Absorption \\
\hline 4 & 3000 & InAlAs & $3 \mathrm{E} 18: \mathrm{Si}$ & Emitter \\
\hline 3 & 5000 & InGaAs & $3 \mathrm{E} 19: \mathrm{Si}$ & Emitter \\
\hline 2 & 1000 & InAlAs & Un-doped & Buffer \\
\hline 1 & 200 & InP & Un-doped & \\
\hline \multicolumn{5}{|c|}{ Substrate SI $:$ InP } \\
\hline
\end{tabular}

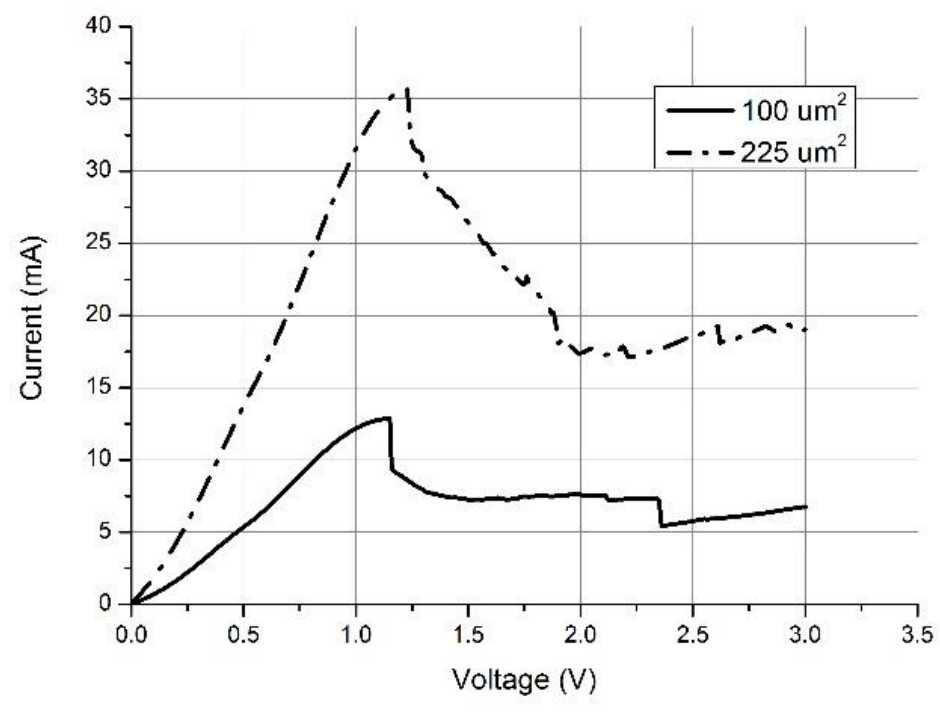

Figure 3. The measured RTD-PD IV characteristics (dark). Two different device size $10 \times 10 \mu \mathrm{m}^{2}$ and $15 \times 15 \mu \mathrm{m}^{2}$ are compared. The negative differential resistance region is between $1.2 \mathrm{~V} \sim 2.4 \mathrm{~V}$.

Table 3. RTD-PD DC characteristics.

\begin{tabular}{|c|c|c|c|c|c|}
\hline Device size $\left(\mathrm{um}^{2}\right)$ & Optical window $\left(\mathrm{um}^{2}\right)$ & $\Delta \mathrm{V}(\mathrm{V})$ & $\Delta \mathrm{I}(\mathrm{mA})$ & $\mathrm{G}_{\mathrm{n}}(\mathrm{mS})$ & Estimated Power $(\mathrm{mW})$ \\
\hline 100 & $3 \times 8$ & 1.2 & 7.4 & 9.3 & 1.7 \\
\hline 225 & $5 \times 10$ & 1.2 & 18.5 & 23.1 & 3.5 \\
\hline
\end{tabular}

Different from normal RTD-PD relaxation oscillator design [4][5][6] with a single RTD-PD device biased in series with a large inductor (transmission line), the proposed RTD-PD LC oscillator circuit utilize two RTD-PDs in parallel, as shown in Fig. 4(c). Each device is biased individually with shunt resistor to suppress the bias oscillation [9]. The schematic circuit of RTD-PD oscillator is as shown in Fig 4 (a), where $R_{e}$ is the stabilizing resistor, $C_{e}$ is the bypass capacitor, $C_{b}$ is the DC block capacitor, $R_{L}$ is the load. The RF equivalent circuit is shown in Fig 4(b). Each RTD-PD is presented by its negative differential conductance $-G_{1 n} /-G_{2 n}$ in parallel with self-capacitances $C_{1 n} / C_{2 n}$. The oscillation frequency is determined by 


$$
f_{o}=\frac{1}{2 \pi \sqrt{\left(C_{1 n}+C_{2 n}\right) L}}
$$

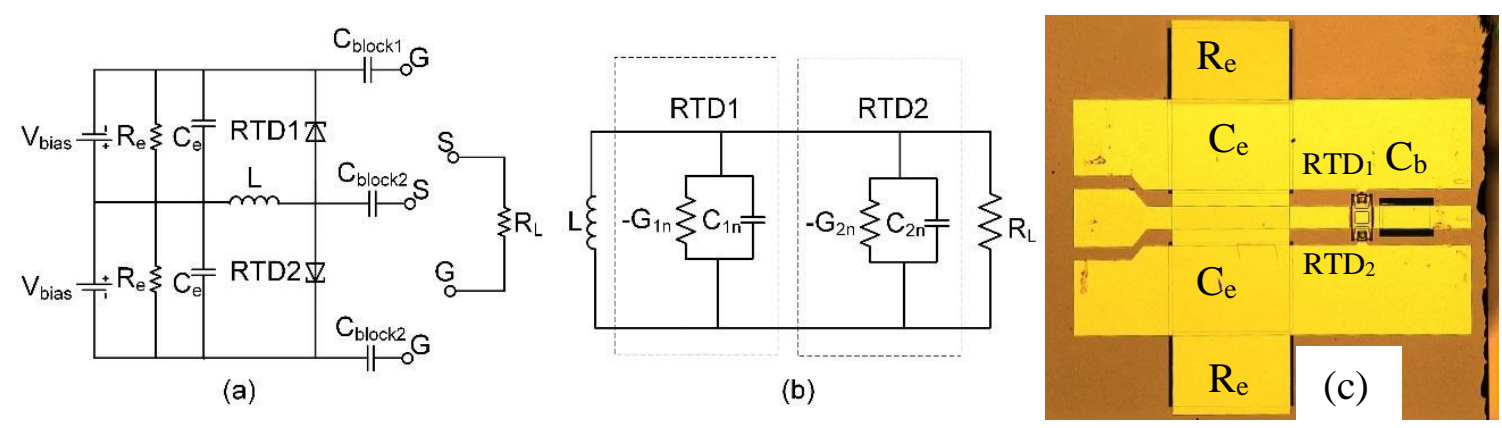

Figure 4. (a) The two RTD oscillator schematic circuit. Each RTD was biased individually with its own DC stabilization circuit $\mathrm{R}_{\mathrm{e}}$ and $\mathrm{C}_{\mathrm{e}}$. (b) RTD oscillator RF equivalent circuit excluding parasitic elements. (c) Fabricated RTD-PD oscillator, $\mathrm{Re}=20 \Omega, \mathrm{C}_{\mathrm{e}}=60 \mathrm{pF}, \mathrm{C}_{b}=7 \mathrm{pF}$.

The fabricated oscillator photograph is shown in Fig 4 (c). $R_{e}$ is thin film NiCr resistor with $R_{e}=20 \Omega, C_{e} / C_{b}$ is metalinsulator-metal (MIM) capacitor with $\mathrm{C}_{\mathrm{e}}=60 \mathrm{pF}, \mathrm{C}_{\mathrm{b}}=7 \mathrm{pF}$. The inductance is introduced by CPW structure. The inductor value is determined by the CPW length. The design procedure can be found in Ref. [9]. To effectively suppress the parasitic oscillation, $R_{e}$ should satisfy that $R_{e}<1 / G_{n}$. From the impedance matching point of view, to obtain the maximum power, the load conductance should satisfy $\mathrm{G}_{\mathrm{L}}=\mathrm{G}_{\mathrm{n}} / 2$. For a $50 \Omega$ load, the optimized device conductance is about $40 \mathrm{mS}$. Referring to Table 2, for $225 \mu \mathrm{m}^{2}$ single device, $\mathrm{G}_{\mathrm{n}}=23.1 \mathrm{mS}$. By utilizing two $225 \mu \mathrm{m}^{2}$ RTD-PD device in parallel, the total conductance is two times larger which is close to $40 \mathrm{mS}$. In this case, $222 \mu \mathrm{m}^{2}$ RTD-PD oscillator provide better impedance matching, the output power is expected high. The measurement results shown in the following section validate the design.

\section{MEASUREMENT RESULTS}

The oscillators were characterized on-wafer by using Agilent E4448A spectrum analyzer. A Cascade ACP65 RF probe was used to probe the output of these oscillators. As the cable loss/system loss is not accurately specified by the manufacturer, the actual power loss was characterized by calibrated another signal source Wiltron 68187B. The measurement results are summarized in Table 4. It shows that high frequency RTD-PD oscillators with over $1 \mathrm{~mW}$ output power have been developed. The frequency can be further increased by optimize the CPW design. The difference between designed and measured frequency is mostly due to the approximation of the device capacitance.

Table 4. RTD-PD oscillator measurement results.

\begin{tabular}{|c|c|c|c|c|c|c|}
\hline $\begin{array}{c}\text { Device } \\
\text { size }\left(\mathrm{um}^{2}\right)\end{array}$ & $\begin{array}{c}\text { Optical } \\
\text { window }\left(\mathrm{um}^{2}\right)\end{array}$ & $\begin{array}{c}\text { CPW length } \\
(\mu \mathrm{m})\end{array}$ & $\begin{array}{c}\text { Designed } \\
\text { Freq(GHz) }\end{array}$ & $\begin{array}{c}\text { Measured } \\
\text { Freq(GHz) }\end{array}$ & $\begin{array}{c}\text { Measured } \\
\text { Power }(\mathrm{dBm})\end{array}$ & $\begin{array}{c}\text { Calibrated } \\
\text { Power }(\mathrm{dBm})\end{array}$ \\
\hline 100 & 24 & 700 & 30 & 20.7 & -6.7 & -1.7 \\
\hline 100 & 24 & 260 & 60 & 34.4 & -6.1 & 0.4 \\
\hline 100 & 24 & 260 & 60 & 37.0 & -7.1 & 0.1 \\
\hline 225 & 50 & 75 & 90 & 44.4 & -7.1 & +1.4 \\
\hline
\end{tabular}

\section{DISCUSSION AND CONCLUSION}

The results presented in the paper show great potential to utilize RTD-PD as high speed optical modulated RF transmitter. The RTD-PD approach to optical/wireless data transmission provides a high efficiency, low cost solution for seamless integration of high speed fiber network with $\mathrm{THz}$ wireless communication. The increased power level makes it feasible for long distance applications. More work such as optical injection locking, optical-wireless data transmission, is currently being done with these RTD-PD oscillators. 


\section{ACKNOWLEDGMENTS}

The authors thank the staff of the James Watt Nanofabrication Centre (JWNC) at the University of Glasgow for help in

fabricating the devices. This work was supported by European Commission, grant agreement no. 645369 (iBROW project).

\section{REFERENCES}

[1] Maekawa, T., Kanaya, H., Suzuki, S., and Asada, M., "Oscillation up to $1.92 \mathrm{THz}$ in resonant tunneling diode by reduced conduction loss," Applied Physics Express, 9(2), 024101 (2016).

[2] Oshima, N., Hashimoto, K., Horikawa, D., Suzuki, S. and Asada, M., "Wireless data transmission of 30 Gbps at a 500-GHz range using resonant-tunneling-diode terahertz oscillator," 2016 IEEE MTT-S International Microwave Symposium (IMS), 1-4(2016).

[3] Figueiredo, J., Romeira, B., Slight, T., and Ironside, C., "Resonant Tunnelling Optoelectronic Circuits," Advances in Optical and Photonic Devices, InTech. ISBN: 978-953-7619-76-3, (2010).

[4] Chahal, P., Morris, F., and Frazier, G., "50 GHz resonant tunneling diode relaxation oscillator," 62nd Device Research Conference, (1), 241-242 (2004).

[5] Brown, E. R., Parker, C. D., Verghese, S., Geis, M. W., and Harvey, J. F., "Resonant-tunneling transmission-line relaxation oscillator," Applied Physics Letters, 70 (21), 2787-2789 (1997).

[6] Cantu, H. I., Romeira, B., Kelly, A. E., Ironside, C. N. and Figueiredo, J. M. L., "Resonant Tunneling Diode Optoelectronic Circuits Applications in Radio-Over-Fiber Networks," IEEE Transactions on Microwave Theory and Techniques, 60 (9), 2903-2912 (2012).

[7] Kim, C. S., and Brandli A., "High-Frequency High-Power Operation of Tunnel Diodes," IRE Transactions on Circuit Theory, 416-525 (1961).

[8] Wang, J., "Monolithic Microwave/millimeterwave integrated circuit resoant tunneling diode sources with around a milliwatt output power, " PhD thesis, University of Glasgow, (2014).

[9] Wang, J., Wang, L., Li, C., Alharbi K., Khalid A., and Wasige, E., "W-band InP-based Resonant Tunnelling Diode Oscillator with Milliwatt Output Power," The 26th International Conference on Indium Phosphide and Related Materials, (2014). 